\title{
Imagix: Multimedia software for evaluating the vividness of movement imagery
}

\author{
Jean F. Fournier
}

Laboratoire de Psychologie Appliquée au Sport, Institut National du Sport et de l'Education Physique, France

Article paru dans : Perceptual and Motor Skills, 2000, vol. 90, pp. 367-370

\section{Summary}

This article presents a new software, Imagix, designed for the evaluation of the vividness of movement-imagery. This multimedia test is comprised of 18 movements in six different psychomotor categories whose vividness of imagery is to be appraised. Imagix has the advantage of offering an evaluation of the vividness of imagery in a normative way. The participant chooses among four films displaying a vividness gradient, which includes contrast and luminosity. The participant is first asked to visualize a movement verbally suggested. The participant is then asked to evaluate the vividness of this mental image by choosing on a computer screen the movie that best corresponds to the vividness of the movement-imagery. The first versions of this freeware (in English and French) are currently being validated.

Mental imagery is one of the techniques used by elite athletes in their mental training. Imagery is commonly defined as "the act of imagining whereby a material picture or image in the mind is scanned as a current event" (Drake, 1996, p. 2). There are visual, olfactory, auditory, kinesthetic, and tactile types of imagery; however, when the sense invoked is only visual, imagery is called visualization. Visualization is a type of imagery that is very common among athletes. Some researchers argue that imagery, ii used to improve performance, should be experienced with images that best approximate reality, using all the senses (Murphy, 1994). Characteristics of a mental image include its perspective (internal and external), its controllability, e.g., the ability to produce a mental picture, or its vividness. Vividness is said to be an important factor of quality imagery, enhancing performance (Marks, 1989). It has often been studied; it is assumed to represent the "realness" of the mental picture. Vividness might be better understood, however, by introducing descriptive factors such as depth perception or whether mental pictures are in color. In applied sport psychology, vividness is also understood as "clarity" on the rating scales of the questionnaires designed to appraise this component of visualization (Isaac, Marks, \& Russell, 1986). To explain the "realness" or "clarity" concept we prefer to use the analogy of the television set: an athlete adjusts the vividness of a mental picture when he tunes in his mind the contrast and the luminosity.

Traditional paper-and-pencil imagery tests use Likert-type scales for appraising vividness. These tests present some weaknesses such as the lack of a norm for the value of an answer. For example, the same answer "vague and dirn" can be chosen by two participants to rate mental pictures of different qualities. Likewise, a different rating can be attributed to a mental picture of similar quality whether it concerns two participants or the same one evaluating the characteristics of his visualization at different rimes. Many pencil-and-paper questionnaires of this kind already exist (Atienza, Balaguer, \& Garcia-Merita, 1994). None of them, however, take into account the interindividual variability of the answers. Recently, Chevalier, Monnier, and Auger (1995) drew from Marmor (1975) to propose a test to control this bias. To evaluate the characteristics of mental images of children, they used four pictures displaying a gradient of clarity. They reported that, while their test had weak psychometric validity, children as young as 6 years old were able to visualize pictures of their own movements. Y et if this test 
suggests a way of limiting the difficulty linked to rating subjective and individual evaluation of a mental picture, the rating scale involves still pictures, not filmed representations of movements.

The goal of this article is to present a software designed for evaluating mental imagery using the basic premise of Marmor (1975). In particular, four pictures were used to define the norm, but a more elaborate test was developed based on movies which should better approximate mental images in motion. In our questionnaire, films correspond to a mentally imagined movement whose vividness is to be evaluated. In this way, we hope to evaluate more precisely the vividness of the visualized movements. We also hope to control the variability bias of the more traditional, individual autoevaluative ratings.

\section{Building Imagix}

We extracted six movement categories from three psychomotor taxonomies (Gentile, Higgins, Miller, \& Rosen, 1975; Konorski, 1967; Le Boulch, 1980): (1) Locomotion, e.g., hopping, (2) Locomotion other than forward, e.g., walking backward, (3) Balance, e.g., standing on one leg, (4) Eye to band, e.g., catch a ball, (5) Eye to foot, e.g., stop a rolling ball with a foot, (6) Torso, e.g., turn at the waist. We then selected three movements in each category on the basis of their simplicity. While these categories may appear biased toward field sports, they permit the most general application. Movies may later be adapted to specific sports. The 18 movements are related to the 18 items of the first version of the test. They were filmed (using an actor in a neutral environment) and digitized to allow computer application. Movies corresponding to each item were altered in contrast and luminosity with Adobe ${ }^{\mathrm{TM}}$ Premiere software to produce three qualities of film for every movement in addition to the original film of best quality. The same settings were used to alter the best film so that each set of four films had the same gradation of vividness. These modified movies were then included in an interactive software, IMAGIX, using the HyperTalk language for programming.

IMAGIX is a HyperCard stack that can be run on Macintosh (including PowerMacs), using the system extension QuickTime (activated), and the Apple TM application HyperCard player. These items are free for Apple ${ }^{\mathrm{TM}}$ Macintosh computers, and there is no need to purchase additional graphics or sound cards. Imagix runs on any Macintosh with a 14-in. color monitor and requires 23 megabytes on the hard disk. Users must allocate 4 megabytes to the HyperCard player.

\section{How to run the computer test}

The first card of the stack requests biographical information such as name, age, sex, and sport of the participant. Ali the information for each participant is exported as a text file and is directly available for quantitative analysis. Once the first screen (card) is filled, only movement of the mouse is required to run the test. Every movement to be visualized is first described by a voice, e.g., "Imagine someone walking backward, from left to right ... once you have finished visualizing the movement, click on the 'next' button at the bottom of this screen"; standard headphones can be used to avoid bothering nearby participants. The participant may replay the audio description as many times as needed before clicking on the "button" to proceed. The next card displays on one screen four films of modified versions of the movement. The films are aligned at the top of the screen, ready to be played, always in the same order. The movie of worst quality is in the upper left corner of the screen, followed by the three others, up to the movie of best quality in the upper right corner. Each film can be played by clicking on a play-button beneath it. This can be done as many times as necessary to make a choice. Once the choice is made, the participant selects a vividness by clicking on the select-button below the movie that best corresponds to his mental image. This type of selection eliminates the necessity of a Likert type scale with a number giving an index of the vividness. 
This process gives three ratings: one corresponding to the vividness of the mental image suggested verbally (from 1 to 4 ), the time spent to visualize, and the time spent to make a decision. The first item (imagine someone walking from right to left) gives the participant an idea of the next 18 films now to be seen_ In each of the films, the same figure always moves in front of a white background.

A pilot study was conducted with 10 participants. The average time to take the test was 20 min. It took an average of $31 \mathrm{sec}$. $(\mathrm{SD}==12 \mathrm{sec}$.) to visualize each movement, $15 \mathrm{sec}$. $(S D==$ $8 \mathrm{sec}$.) to play the movies, and $17 \mathrm{sec}$. $(\mathrm{SD}==8 \mathrm{sec}$.) to choose among the four movies. For this sample of participants, the mean of the vividness score was $3.0(\mathrm{SD}==0.9)$. Participants reported that the four movies of the first item laid the "basis" for the other items of the test. They understood that their choices would consist of four movies depicting a gradient of vividness and that they would have to choose among movies which presented the same actor in the same environment. The first item was therefore helpful to standardize in their imagery not only the background (white) but also the actor (male character, wearing blue jeans and a brown sweater) for the movements remaining to be visualized.

The two current versions (English and French) are available from the author, for research purposes only. IMAGIX is free; only distribution costs are required. This multimedia test is currently being validated. In the interest of enhancing bath validation and the actual test, we encourage researchers to send us raw, anonymous data.

\section{Future research}

We created IMAGIX to promote a more objective way of appraising one characteristic of mental pictures. The same stack can serve as a basis for evaluating other characteristics of mental pictures such as auditory imagery by modifying the rhythm in the movies offered for selection. Another way of adapting IMAGIX to another parameter of imagery would be to use movies of similar clarity played at different speeds. In addition, different perspectives (internal or external) could be offered as choices to the participants. IMAGIX could also be adapted to the experience and skill of the athlete or to the specificity of a sport, e.g., with close-ups in a gymnasium or with a panoramic view of a football match.

\section{References}

ATIENZA, F., BALAGUER I. \& GARCIA-MERITA, M. L. (1994) Factor analysis and reliability of the movement imagery questionnaires. Perceptual and Motor Skills, 78, 13231328.

CHEVALIER, N., MONNIER, E., \& AUGER, R. (1995) L'image visuelle des mouvements corporels de J'enfant de six ans [Visual images of body movements in six-year-old children]. Sciences et Techniques des Activités Physiques et Sportives, 16, 36, 57-66.

DRAKE, S. M. (1996) Guided imagery and education: theory, practice and experience. Journal of Mental Imagery, 20, 1-58.

GENTILE, A. M., HIGGINS, J. R., MILLER, E. A., \& ROSEN, B. M. (1975) The structure of motor tasks: movement. [Special Issue] Proceedings of the Seventh Annual Canadian PsychoMotor Learning and Sports Psychology Symposium. Montreal: APAPQ. Pp. 11-28.

ISAAC, A., MARKS, O. F., \& RUSSELL, O. G. (1986) An instrument for assessing imagery of movement: the Vividness of Movement Imagery Questionnaire (VMIQ). Journal of Mental Imagery, 10, 23-30. 
KONORSKJ, J. (1967) Integrative activity of the brain: an interdisciplinary approach. Chicago, IL: Univer. of Chicago Press.

LE BOULCH, J. (1980) L'éducation par le mouvement: la psychocinétique [Education by the mouvement]. Paris: Editions Sociales Françaises.

MARKS, D. F. (1989) Bibliography of research using the vividness of visual imagery questionnaire. Perceptual and Motor Ski//s, 69, 707-718.

MARMOR, G. S. (1975) Development of kinetic images: when does the child first represent movement in mental images? Cognitive Psychology, 7, 548-559.

MURPHY, S. (1994) Imagery intervention in sport. Medicine and Science in Sports and Exercise, 26, 486-494.

The author thanks Marc Guastavino for his assistance with the programming of the software and Eric Monnier for his film performance 